\title{
THE DENTAL OFFICERS' ROLE - IN A DISASTER SITUATION *
}

\author{
Major J. D. FLEET, M.B., B.S., F.D.S., R.C.S., R.A.D.C. \\ Cambridge Military Hospital, Aldershot
}

\section{Introduction}

IN considering further the role of the dentist in a disaster situation, I would like to consider his function in a field ambulance, or field hospital or in the case of civilian disasters, his role in the local casualty hospital.

Why can the dentist be of value? What roles can he perform?

Previous writings on the subject have suggested that the dentist is capable of being used for a wide, range of different tasks from triage of casualties to acting as emergency midwife.

I would suggest that his functions should in the first instance be threefold:-

a. As a member of the resuscitation team. b. As an aid to the anaesthetic staff. c. The treatment of dental and maxillo facial injuries.

If subsequently he had little function in any of these roles due to a decrease in the number of casualties, his knowledge will direct him to other roles he can play; for example, aiding the physicians, surgeons, administrators, or treating minor injuries.

It is important that the dentist realises that in any disaster situation he is going to have to play a leading role in providing medical assistance. It is essential that he receives training to carry out these functions and has regular opportunities to practice the techniques so as to be prepared for a disaster situation.

Let us consider these roles:

\section{Resuscitation}

Why is the dentist suited to this role?

a. He has studied physiology and has a knowledge of body fluids and the pathophysiology of shock.

b. He has the knowledge of anatomy and with a little extra training can be taught the anatomy of the important sites in the arms and legs for intravenous cannulation.

c. Many dentists are already familiar with venepuncture techniques using them in everyday dentistry for induction of anaesthesia with short acting barbiturates, or using them for tranquilliser drugs like Diazepam.

Let us briefly review the key facts he needs to know in the treatment of shocked patients and then consider the techniques needed to act as a resuscitation officer.

Shock is a state of reduced tissue perfusion which leads to generalised cellular hypoxia and vital organ damage. It can occur due to a decrease in circulating blood volume as in haemorrhage or burns. It can occur due to an increase in the capacity of the vascular system as in septic, anaphylactic, and neurogenic shock. Lastly, it may occur due to failure of the pump itself in cardiogenic shock. 
In this presentation we will confine ourselves to hypovolaemic shock being the variety of shock most likely to be seen in a disaster situation. The dentist should however be aware of the other varieties of shock and in the course of his training have some knowledge of their treatment.

In shock there is a decreased venous return to the heart which leads to a fall in cardiac output and consequently to a decreased perfusion of the tissues by the blood. This results in decreased oxygenation of the tissues and damages vital organs like the kidney, brain, heart and liver.

The ultimate damage in shock is due to cellular hypoxia. As Lord Haldane said "Anoxia not only stops the machinery, it wrecks it".

Of course the body has its own response to shock, acting via the sympathetic nervous system:

a. Increased peripheral arteriolar tone decreases perfusion of non-essential organs, like the skin and gut.

b. Increased venomoter tone squeezes blood out of the large venous reservoirs into the circulation. As 80 per cent of the blood volume is in the veins this mechanism can compensate for a 10 per cent loss of blood volume without any fall in blood pressure.

c. Increased sympathetic drive to the heart increases heart rate and cardiac output.

The sympathetic responses being maximal in skin, gut, muscle mass and kidney divert blood away from these organs and may prove damaging to them in the long term. Clinically the patient with shock presents with the classical signs as presented in any First Aid manual.

In a young fit patient the defence mechanism of the body is so effective that the blood pressure may not only be maintained but may actually rise initially in response to blood loss. However, the vasoconstriction needed to maintain the blood pressure will lead to stagnant hypoxia and vital organ damage.

The essential principle of treatment of shock is to secure the blood flow. "To turn the swamp into a running brook". This is best achieved by intravenous infusion and is well illustrated by considering hypovolaemic shock.

\section{Hypovolaemic shock}

This will be common in any disaster situation. It occurs due to:-

a. Haemorrhage. b. Fluid loss in burns and crush injuries. c. Trauma, where blood and fluid loss occurs in and around injured parts.

It is essential to be aware that a patient can die of hypovolaemic shock without any obvious blood loss due to fluid loss into fractures. The dentist should have some rough ideas of assessing blood loss:

Wound the size of a closed fist $=\frac{1}{2}$ litre of fluid. Surface wound the area of a hand $=\frac{1}{2}$ litre. Fractured femur $=$ at least $\frac{1}{2}$ litre. Fractured pelvis $=$ at least 1 litre.

\section{Haemorrhagic shock}

In order to survive, the tissues need oxygen and this is carried by the haemoglobin. A bountiful Maker provided us with excess haemoglobin to our needs and it is quite safe to operate on people with a haemoglobin of $10 \mathrm{~g} / 100 \mathrm{ml}$, that is 70 per cent normal. 
In haemorrhagic shock the patient has not only lost haemoglobin but because of slow blood flow in the capillaries he gets sludging of the red blood cells, further lowering the concentration of red cells in his general circulation and further decreasing his haemoglobin concentration.

So in treating these patients the essentials are, to stop the haemorrhage and then to get the circulation going with any available compatible fluid. In this way the sludging of red cells is prevented and these cells are made available for oxygen transport.

The most important thing is Blood Flow and it is lack of this which is fatal rather than the low haemoglobin concentration.

The same principles apply in treating burns casualties. Due to plasma loss into blisters and out of raw areas haemoconcentration occurs. Fluid must be replaced to secure blood flow and prevent hypovolaemic shock.

Ideally this should be by the intravenous route, but in a disaster situation it is essential to appreciate, as pointed out by Boulton and Cole (1966), that up to 30 per cent burns can be treated with oral fluids, using half-normal saline-bicarbonate solutions. This may be the only route for the majority of patients when one considers that the number of casualties to be expected if a large bomb fell on a major city may be about 200,000 people (Drummond 1957).

There are many formulae for calculating the fluid requirement but the best method is frequent clinical assessment. If the pulse rate continues to rise, if the blood pressure falls, or if urine output is less than $50 \mathrm{ml} /$ hour, more fluid is urgently needed.

For disaster situations the ideal solution to be stockpiled and made available should be cheap and easy to store at any temperature. It should be in robust cartons and be sterile and isotonic with normal blood.

The ideal solutions are balanced salt solutions like Hartmann's or Ringer lactate which contain electrolytes in approximately the same concentrations as plasma. Alternatively normal saline, Dextrose saline or Dextrose-solutions can be used.

Dextrans may be available. These polysaccharide polymers of molecular weight 70,000 to 110,000 leave the vascular spaces less readily than the simple electrolyte solutions mentioned above, and hence act as plasma expanders. They are excellent in the treatment of shocked patients but they are expensive and their storage is more critical as regards temperature.

Blood and plasma are likely to be in short supply and the facilities for collection, screening for infection, cross-matching and storage in refrigerators makes them unlikely to meet the demands of a disaster situation. In any case, as Crocker (1968) pointed out it is doubtful if a severely shocked patient is benefited by the rapid transfusion of several litres of cold blood with all the risks of a blood transfusion under less than ideal conditions:

So in patients with hypovolaemic shock, the dentist acting as resuscitation officer must remember that it is blood flow which is important, and that an infusion of a simple electrolyte solution will give the patient the best chance of survival. He must remember the measurable parameters of shock available even in a disaster situation:-Pulse, blood pressure, respiratory rate, fluid input and output.

The blood pressure may offer little guide as mentioned earlier because a fit man may loose 30 per cent of his blood volume without a significant fall in blood pressure. 
Tachycardia is usually a more sensitive indicator of blood loss than is blood pressure measurement; a rising pulse indicating a need for more fluid.

Central venous pressure measurement is the ideal and must be known about by anyone dealing with shocked patients, but it is unlikely to be available in a disaster situation. However, as Wolfson (1962) describes, examination of the veins on the dorsum of the foot with the patient horizontal gives a good indication of the circulatory status. Jugular venous filling can also be used as these veins also fill when the circulatory volume is restored to 70 to 80 per cent of normal. Adequacy of circulatory volume can also be assured by monitoring urinary output and if $1 \mathrm{ml} / \mathrm{min}$ is excreted by an adult then the patient is in fluid balance.

From a training point of view he must know the anatomy of the veins of the arm and leg and the usual sites for intravenous cannulation.

He must practice cannulation with the usually available types of cannula.

He must know the fluids usually available for infusions and be aware of the problems of any infusion, for example, thrombophlebitis, fever, allergic reactions, air embolism and heart failure due to circulatory overload.

The problems of blood transfusion need special emphasis, for example, incompatability, infection, hyperkalaemia, hypocalcaemia, hypothermia. From this it can be appreciated that blood can be a most dangerous treatment for a patient and especially when conditions are less than ideal as in a disaster situation.

In conclusion the dentist must be trained-To recognise the types of shock; to understand the measurable parameters of shock; to realise that the basic problem in shock is one of blood flow, best treated by intravenous infusion of a simple electrolyte solution; to understand the complications of shock as well as those of intravenous infusions.

With this training the dentist can then be prepared to act usefully in resuscitating the vast numbers of patients to be expected in a disaster situation.

\section{The dentist as an anaesthetist}

In any disaster situation so great will the number of injured people be that, for the emergency, it will be necessary to use acting anaesthetists as well as qualified anaesthetists.

The most logical first choice of person for acting anaesthetist is the dentist because:-

a. He is trained to give anaesthetics for dental purposes. b. He understands the principles of obtaining a clear airway and maintaining it. c. He understands the physiology of respiration. d. He knows the anatomy of the respiratory tract. e. He knows the pharmacology of most of the agents used. f. He knows the medical conditions affected by general anaesthetics. g. He is capable and practised at intravenous injections, many dentists using them regularly in dental practice.

The additional knowledge he needs is as follows:-

a. Training in endotracheal intubation. $b$. Knowledge of muscle relaxant drugs and how to reverse their activity. c. Training in the practice of anaesthesia and especially under different conditions. 
This latter feature is especially important for military dentists who may go out with a field unit to a disaster situation and have to practice anaesthesia under the most difficult conditions.

Military anaesthesia probably began when ether was used on the battlefield in 1847 during the Mexican War and stimulus to progress in anaesthesia came from both World Wars-an example of good coming out of evil.

We are now in an area of highly specialised anaesthetic skills and techniques but we must evolve a technique suitable to the occasional emergency anaesthetist working under the most primitive conditions.

In any disaster situation, both military and civilian, the supply of bottled anaesthetic gases may not be available. They are bulky, heavy and logistically unsound because they provide relatively few anaesthetic hours in proportion to their bulk. Their supply may be non-existent especially with nitrous oxide which is only manufactured by a few highly industrialised countries. Oxygen may be more easily available because of its use for non-medical purposes, like oxyacetylene tools and if so it can be used for air-enrichment in the anaesthetic technique discussed below.

Because of these transport and supply difficulties it is essential to evolve a basic simple technique, of use in the most primitive conditions, and then, if by chance more sophisticated facilities are available then the occasional anaesthetist can easily rise to them.

The technique envisaged uses a volatile anaesthetic agent in a vapouriser using air as a carrier "gas" and a resuscitatory bag like the Ambu bag as a ventilator as described by Boulton and Cole (1966). There are several volatile agents which could be used.

Ether is safe, universally available and cheap but because it is highly volatile a large quantity is needed for each patient and as it is highly inflammable, aircraft captains may refuse to carry it.

Chloroform is cheap, produces satisfactory anaesthesia but as it is a very potent agent it needs great skill and care in its use and its storage in glass jars is a drawback from a transport point of view.

Halothane is safer than chloroform but it is also stored in glass bottles. It is very volatile, highly expensive and when used with air needs a high concentration to be an efficient analgesic agent.

Provided muscle relaxants and the means of intubation and ventilation are available trichlorethylene (Trilene) is the volatile agent of choice. It is safe, cheap, non-inflammable, a good analgesic with high logistic soundness because one tin of Trilene containing $250 \mathrm{ml}$ will provide anaesthesia for 50 operations of 1 to 2 hours duration.

So having decided on the agent that our emergency anaesthetist will use it is necessary that he knows a standard technique applicable under field conditions. In the light of extra facilities being available he can then modify the scheme.

a. First he must assess the patient's general medical condition, looking for conditions which would make general anaesthesia unsafe.

b. Then he must ensure proper pre-operative preparation of the patient. 
Especially important is the treatment of shock by fluid replacement, and the need for pre-operative abstinence from food and drink, remembering that gastric emptying is delayed in shock patients.

c. Premedication of the patient with atropine is advisable.

d. An intravenous cannula must be inserted into a vein prior to induction to give ready access to the circulation for treatment of any anaesthetic or operative complications.

e. Induction is then carried out using intravenous thiopentone or methohexitone. A depolarising muscle relaxant like suxamethonium (Scoline) is then given and the patient briefly ventilated with air or oxygen if available.

f. An endotracheal tube is then passed and the patient ventilated with the Trilene/ air mixture (plus oxygen if available). Intubation guarantees a safe airway and under difficult conditions is probably safest for the patient.

g. Muscle relaxation is continued using a non-depolarising relaxant like gallamine (Flaxedil) injected intravenously as required to allow continued ventilation with the Trilene/air mixture. If the patient was not paralysed but allowed to breath the Trilene/ air mixture spontaneously he would Not receive the required 1 per cent Trilene essential for analgesia.

h. Now the dentist must monitor the patient throughout the operation. Monitoring pulse and respiration and replacing blood loss with blood if available, otherwise by simple electrolyte solutions as discussed above in order to maintain the blood pressure

This monitoring is essentual with gaseous agents like Trilene as, if, signs of overdosage occur, as evidenced by cardiac irregularities, respiratory failure or cyanosis the vapouriser can be disconnected and by ventilating with air the excess. Trilene can be dissipated.

j. At the termination of the operation the muscle relaxant can be reversed, in the usual way using intravenous neostigmine preceded by intravenous atropine to reduce the cardiac effects.

k. When spontaneous respiration returns the patient can be extubated and placed in the coma position and then carefully observed till full recovery occurs which is usually rapid using Trilene and muscle relaxants. Rapid recovery is essential to any technique in a disaster situation when skilled recovery room personnel are going to be in short supply.

Some authorities favour intermittent intravenous thiopentone or mexohexitone but for the occasional anaesthetist like the dentist in a disaster situation overdosage is fatally easy to perform. Like salt in your soup you can always add intravenous agents agents but they are very difficult to take out again.

So I have made out a case for the dentist to be taught a standardised technique using inhalational anaesthetics because it fulfills the three objects of anaesthesia:

a. To keep the patient alive throughout surgery. b. To make surgery painless. c. To provide the best possible operating conditions.

To complete his role the dentist must also be prepared for the complications of anaesthesia and be taught how to pass a naso-gastric tube to empty the stomach and so 
avoid the danger of inhalation of vomit. He must know how to deal with laryngospasm, cardiac arrest etc.

It is desirable that the dentist should have an intensive 2 or 3 week training by an experienced anaesthetist in a busy hospital to gain experience in the techniques discussed. He should also spend at least half-a-day a month keeping the techniques refreshed by working in an anaesthetic department. This will not only keep him prepared for a disaster situation, but the knowledge gained will make for greater safety of his patients in his regular dental practice.

Remember forearmed is forewarned and proper training allows anticipation of complications and teaches the correct ways of maintaining a clear airway in order to ensure adequate oxygenation. This will then avoid the Black Death - the plague of Anaesthesia.

\section{The dentist as a dental and maxillofacial expert}

Every dentist working in a disaster situation is obviously able to perform the basic dental procedures necessary to deal with simple dental problems complicating a patient's condition. But in a disaster situation, be it military or civilian he needs to have a working knowledge of the principles of treatment of maxillofacial injuries, and be able to commence emergency treatment prior to evacuation of the patient to specialist care.

In previous military disasters the incidence of maxillofacial injuries was about 10 per cent due to the effects of missiles on the exposed head. In a natural disaster the incidence will be lower than this.

Experience in the Second World War, Korea and Vietnam have emphasised the good results obtained by early operation and closure of facial wounds, together with greater use of antibiotics. Early definitive operations made possbile by speedy helicopter evacuation to specialist units offered the best possible prognosis for head injury casualties.

However none of the excellent back-up facilities are of use if the first person with any maxillofacial experience fails to use it for the safety and benefit of the patient.

The dentist working in a disaster situation must understand the principles of treatment of maxillofacial injuries:

Preservation of life. Maintenance of function. Restoration of appearance.

He must be trained to approach these patients in a standard way and be able to commence early management as described by Rowe and Killey (1968).

a. On a conscious patient, a history must be obtained of the date and time of the injury and of any treatment received. A knowledge of the type of trauma and direction of force may be helpful.

It is essential to determine if there has been any loss of consciousness and the extent of possible cerebral damage. Patients who have lost consciousness must be carefully monitored in case of any rise in intracranial pressure due to haemorrhage.

Patients with head injuries who have lost consciousness must have regular monitoring of consciousness level, pulse, blood pressure and pupillary reactions. Deteriorating consciousness, a rising blood pressure, a falling pulse rate, or a dilating pupil indicating an urgent need for neurosurgical intervention. 
It is also important to ascertain from the patient whether he was wearing any prosthetic appliance, and if so its whereabouts should be determined, and if not found remember that it may be in the soft tissues, trachea, lungs or oesophagus.

b. A general examination is essential to exclude other and more serious injuries, and attention should be directed to the thorax, abdomen and limbs for a possible cause.

c. A thorough extra-oral and intra-oral examination must be carried out. Careful palpation of the bony skeleton before the effects of oedema mask signs of fractures may be as accurate as any X-ray which is unlikely to be available in a disaster situation.

d. Careful examination of the nose and ears must be made for cerebrospinal fluid (c.s.f.) leakage.

e. Initial treatment both general and local can then be commenced.

\section{Respiratory obstruction}

\section{General Treatment}

This may occur due to inhalation of blood clot, teeth, dentures or due to loss of support of the tongue in mandibular symphyseal fractures. It can also occur in maxillary fractures when retroposition of the middle third of the face allows the soft palate to obstruct the oro-naso-pharynx.

Respiratory obstruction can be overcome by suction of the oro-pharynx and forward positioning of the tongue either by lying the patient prone, or by using an oropharyngeal airway, or by endotracheal intubation.

In extreme cases tracheostomy may be required. Kwapis (1954) in his review of maxillofacial war injuries in Korea claimed that the mortality rate of zero which he obtained was due to judicious use of tracheostomy in cases with severe respiratory obstruction.

This was also borne out in Vietnam where elective rather than emergency tracheostomy'was used to relieve existing or potential airway obstruction.

In middle third injuries to the face the simple expedient of digitally pulling the middle third forward relieves the oro-naso-pharyngeal obstruction.

\section{Haemorrhage}

This is rarely a problem and can be treated by ligature of obvious bleeding vessels otherwise by packing of the wound with gauze swabs.

\section{Shock}

This is rarely severe in maxillofacial injuries unless gross soft tissue damage occurs and can be dealt with by intravenous infusions as discussed above.

\section{Infection}

This is the keystone of successful treatment of maxillofacial injuries and the early use of antibiotics, when available, and in the case of fractures with c.s.f. leakage, the use of sulphonamides ensures a high success rate in the treatment of these injuries.

As regards tetanus-this is rare in the face because of the excellent blood supply, but the dentist dealing with wounded people where dirt contamination is likely should be aware of the need for prophylaxis as described by Laurence (1973). If people have 
been regularly immunised with tetanus toxoid, the only treatment required is thorough wound debridement to eliminate any nidus of infection where the anaerobic organisms can flourish. The patient can then be given an antibiotic such as penicillin and a booster dose of tetanus toxoid.

However where no previous immunication has occurred the patient needs tetanus antitoxin in addition to wound debridement, antibiotics and the commencement of tetanus immunisation with tetanus toxoid.

Animal experiments have shown that a lethal dose of toxin can be produced in four hours. Any patient who has untreated contaminated wounds four hours or more old, who has not been previously immunised must be given tetanus antitoxin to neutralise any formed toxin. Since the antitoxin is an animal protein there is a risk of allergic reactions and, ideally, a test dose subcutaneously should be given prior to the full dose to test for allergic response. In sensitive patients, human antitoxin should be given if it is available.

Pain

This can be treated by early immobilisation if only of a temporary nature and by the use of analgesics, always remembering that opiates may further depress an embarrassed respiration, and by their miotic effects mask the pupillary signs of raised intracranial pressure.

The use of a head bandage or a simple horizontal wire between teeth across the fracture site, or an intermaxillary wire between the intact symphysis and the maxilla, may be used for temporary immobilisation for relief of pain.

Intermaxillary fixation should not be used for patients with severe soft tissue damage to the tongue because the inevitable oedema may cause respiratory obstruction.

\section{Local treatment}

The dentist can remove debris such as loose teeth and broken dentures from the mouth, always remembering to preserve broken dentures and send them with the patient as an aid to later treatment for determining the occlusion or for use as splints. Wound toilet can be carried out preserving bone, teeth and soft tissues whenever possible.

Kwapis (1954) in his review of treatment in Korea compared to the Second World War showed that all bone whether attached to soft tissues or not should be preserved and reinserted, as the use of antibiotics and drains made sequestration a rare occurrence. This policy has also been used in Vietnam with similar success.

In the event of major soft tissue loss the simple expedient of suturing skin to mucous membrane protects the bone from infection and preserves tissues till the patient can be transferred to a specialist unit.

Temporary immobilisation of fractures can be carried out, the great emphasis being on simple techniques such as eyelet wires, arch bars and head bandages. Intermaxillary fixation is best carried out using elastic ligatures, so that if the airway becomes a problem during evacuation they can easily be cut by unskilled personnel. In the case of maxillary fractures, the dentist can help most by digitally pulling forward the displaced maxillary segment to improve the airway, and by arranging for the patient to be transported to a maxillofacial specialist in a prone or semi-prone position. 
In the early treatment of patients with maxillofacial injuries the emphasis is on preservation of life, definitive treatment being delayed only as long as it takes for the patient to be evacuated to a specialist unit. There seems to be no doubt that in the case of severe maxillofacial defects especially of the gun-shot wound type, that the secondary objects of treatment, maintenance of function and restoration of appearance, are best achieved if the patient is treated early. This can be achieved either by rapid helicopter evacuation to specialist centres, or by the forward positioning of specialist maxillofacial surgeons.

\section{Conclusion}

In conclusion, the emphasis in this section is on the dentist being trained in the initial assessment of maxillofacial injuries. He should be competent to attend to lifesaving procedures, understand about simple immobilisation techniques, and be able to plan for the patient's safe transfer to specialist treatment.

Finally, one must appreciate that in any disaster situation less than 10 per cent of the injured will have maxillofacial injuries, so the dentist must be trained and prepared for his major roles in the resuscitation of shocked patients, and as an anaesthetist able to use basic techniques for the emergency operations which so many people are going to need.

\section{Acknowledgements}

I would like to acknowledge the great help given to me in the preparation of the section on Anaesthesia by Lieutenant-Colonel D. S. Hutton, M.B., B.S., F.F.A.R.C.S., M.R.C.S., L.R.C.P., R.A.M.C.

I am grateful also to Brigadier E. D. Stanhope, M.B.E., F.D.S., H.D.D., R.C.S. and Major T. R. Peacock, B.D.S., F.D.S., R.C.S., R.A.D.C. for their helpful criticisms.

\section{REFERENCES}

Boulton, T. B. and Cole, P. V. (1966). Anaesthesia 21, 397 and 268.

Drummond, A. (1957). Proc. roy. Soc. Med. 50, 967.

KWAPIS, B. W. (1954). J. oral Surg, 12, 293.

LaUrence, D. R. (1973). Clinical Pharmacology. Churchill Livingstone. Edinburgh and London.

Rowe, N. L. and KILLeY, H. C. (1968). Fractures of the Facial Skeleton. E. \& S. Livingstone, Edinburgh.

WolfSON, L. J. (1962). Anaesthesia for the Injured. E. \& S. Livingstone, Edinburgh. 